\title{
Damage in impact fragmentation
}

\author{
N. Sator $1^{1} \star$ and H. Hietala \\ * Laboratoire de Physique Théorique de la Matière Condensée, Université \\ Pierre et Marie Curie (Paris 6), UMR CNRS 7600 - 4 place Jussieu, 75252 \\ Paris Cedex 05, France, \\ $\ddagger$ Department of Physics, University of Helsinki - P.O.Box 64, FI-00014 \\ University of Helsinki, Finland
}

February 22, 2019

\begin{abstract}
Using a simple and generic molecular dynamics model, we study the damage in a disk of interacting particles as the disc fragments upon impact with a wall. The damage, defined as the ratio of the number of bonds broken by the impact to the initial number of bonds, is found to increase logarithmically with the energy deposited in the system. This result implies a linear growth with damage for the total number of fragments and for the power law exponent of the fragment size distribution.
\end{abstract}

PACS numbers: $46.50 .+\mathrm{a}, 62.20 . \mathrm{mm}$

Keywords: fragmentation, damage, computer simulation

\section{Introduction}

Because of its importance in natural phenomena and industrial processes, fragmentation is of great interest. To a certain extent, fragmentation phenomena depend on the particular features of the object that is broken. However, some generic behaviours seem to be shared by fragmenting systems whatever their size, material, or typical interaction energy. For instance, as observed in a large variety of experiments 1, 2, 3, 4, 5, 6, 7, 8, 9, 10, and natural phenomena [1, 11, 12, the fragment size distribution frequently exhibits a power law behaviour, the origin of which is still unknown.

Despite its simple and rather everyday aspect, fragmentation is a complex and distinctly far-from-equilibrium process which is beneficially studied with computer simulations [13, 14, 15, 16, 17, 18, 19, 20, 21, 22, 23. Indeed, this approach can grasp the complexity and the dynamical properties of fragmentation by taking into account the various parameters that may influence the process — and consider them one by one.

With this aim in mind, we recently proposed an elementary molecular dynamics (MD) model 23] which, due to its simplicity, provides a very generic frame of reference for fragmentation studies. We then applied it to investigate the fragmentation of a two-dimensional disc of interacting particles upon impact with a wall 23 . One of the main results of this study is, that the power law

\footnotetext{
${ }^{1}$ sator@lptmc.jussieu.fr
} 
exponent of the fragment size distribution increases logarithmically with the energy deposited in the system. This behaviour is in agreement with experimental results [2, 10] and we expect it to be generic for fragmentation phenomena.

In the present paper, we extend this study by addressing the issue of damage occurring in a fragmenting disc. In particular, we will show how the breaking of bonds at the microscopic level is related to macroscopic quantities such as the fragment size distribution. Dynamical quantities dealing with the spreading of damage during the fragmentation process will also be discussed.

The paper is organized as follows: in section 2, we describe the model and the simulation procedure ; in section 3 we review the main results concerning the fragment size distribution ; section 4 is devoted to the study of damage ; in section 5 we discuss the fragmentation energy. We conclude in section 6 .

\section{Model and simulations}

The model of fragmentation we use in the present study was described in detail in the previous article 23. A brief summary is presented here.

The fragmenting disk is made up of $N=1345$ particles placed on a twodimensional triangular lattice. The cohesion of the system is ensured by a central two-body Lennard-Jones type of potential

$$
\mathrm{v}\left(\mathrm{r}_{\mathrm{ij}}\right)=v_{0} \epsilon\left[\left(\frac{\sigma}{\mathrm{r}_{\mathrm{ij}}}\right)^{a}-\left(\frac{\sigma}{\mathrm{r}_{\mathrm{ij}}}\right)^{b}\right] .
$$

Here $\mathrm{r}_{\mathrm{ij}}$ is the distance between particles $i$ and $j$ and the two constants, $\epsilon$ and $\sigma$, are the depth of the potential well and the diameter of the particles, respectively. The three parameters, $v_{0}=107.37, a=80$ and $b=78$, were chosen to obtain a very short range of attraction (around $0.1 \sigma$ in addition to the particle diameter). Note that the behaviour of the system under fragmentation is not sensitive to a particular choice of the parameters $v_{0}, a$, and $b$, that is, to the range of attraction [23].

The fragmentation process was studied by means of 2D molecular dynamics simulations utilizing the Verlet algorithm [24, 25] at constant energy. The time step is chosen as $\delta t=0.0001 t_{0}$, where $t_{0}=\sqrt{\epsilon \sigma^{2} / m}$ is the unit of time and $m=1$ the particle mass. In the following, times, velocities and energies are expressed in the units of $t_{0}, \sigma / t_{0}$, and $\epsilon$ respectively.

The disc of diameter $L=40 \sigma$ is constructed, rotated by a random angle and launched into a solid wall with a given impact velocity $\mathbf{V}$. The particles of the disc interact with the wall through the repulsive part of the potential $v\left(r_{i j}\right)$ (for details, see 23). For each value of the impact velocity, we performed 1000 runs while sampling the initial angle of rotation uniformly. Simulations were stopped at $t=100 t_{0}$ (i.e. after $10^{6} \mathrm{MD}$ time steps), when the fragmentation process had already reached a steady state $[23$.

During the simulation, fragments are defined as self-bound clusters of particles [26. In other words, two particles are bonded if their relative kinetic energy is lower than the absolute value of their interaction energy. Once the distance $r_{i j}$ between two particles $i$ and $j$ becomes larger than the attractive range $(\sim 1.2 \sigma)$ of the potential, their potential energy is set from $\epsilon$ to $0.001 \epsilon$. This value reduces the interaction between these two particles to an almost repulsive one and thus the possible recombination is prevented. 
In this article, we measure the energy deposited in the system with a dimensionless control parameter $\eta$, defined as the ratio of the initial kinetic energy to the potential energy of the disc:

$$
\eta=\frac{m V^{2}}{2\left|e_{\text {pot } 0}\right|} .
$$

Here $e_{\text {pot } 0}=-2.87 \epsilon$ is the initial potential energy per particle in the disc. When the kinetic energy is large enough compared to the cohesion of the disc, fragmentation occurs.

The same numerical experiment - a disc of interacting particles fragmenting upon impact with a wall - has been previously investigated by means of more complex models. For instance, Thornton and his collaborators [17] used primary particles interacting through autoadhesive and frictional forces that act as functions of contact area. The particles, set initially in random locations, are pulled together by applying a centripetal force. Hence in their model, the fragmenting agglomerate has an irregular shape and contains inherent structural defects. Likewise, in the model proposed by Kun, Herrmann, and their collaborators [19, 20, 21, the primary particles are rigid randomly shaped convex polygons. These polygons are connected with beams that take into account elastic, shear, and torque interactions.

The asset of the present study is the use of a minimal fragmentation model since it provides a generic frame of reference to which one can compare the effects of different parameters.

\section{$3 \quad$ Fragment size distribution}

For a given amount of energy deposited in the system, we calculated the fragment size distribution - the number $n(s)$ of fragments made up of $s$ particles - averaged over 1000 runs. In addition to this, we calculated the normalized mean size of the largest and the second largest fragment $-S_{\max 1}$ and $S_{\max 2}-$ as well as the total number of fragments $m_{0}$. These last quantities are plotted in Fig. 1 as functions of $\eta$ at the end of the simulation $(t=100)$. As we found in the previous study [23], $S_{\max 1}$ decreases with $\eta$, whereas $S_{\max 2}$ is peaked at $\eta_{t}=0.19\left(V_{t}=1.05\right)$. When the amount of energy deposited in the system is larger than this threshold value $\eta_{t}$, the fragment size distribution exhibits a power law behaviour with an exponent $\tau: n(s) \sim s^{-\tau}$.

To estimate the exponent $\tau$, we employed statistical procedures proposed by Clauset et al. [27, which are based on the method of maximum likelihood. Note that, as $\eta$ increases to values much larger than $\eta_{t}$, the power law region of $n(s)$ narrows due to the finite size of the fragmenting disc, and the determination of $\tau$ becomes difficult. Nevertheless, the power law fit is quite good for $\eta_{t} \leq \eta \leq 4$, (see the fragment size distribution plotted in the inset of Fig. 2 ).

Furthermore, as illustrated in Fig. 2, the exponent $\tau$ of the power law exhibits a logarithmic increase with the energy deposited in the system:

$$
\tau=\alpha_{\tau} \ln \frac{\eta}{\eta_{t}}+\beta_{\tau}
$$

where $\alpha_{\tau}=0.26 \pm 0.01$ and $\beta_{\tau}=1.34 \pm 0.01$. When $\tau$ is given as a function of the impact velocity, the coefficients $\alpha_{\tau}$ and $\beta_{\tau}$ are slightly different than those 


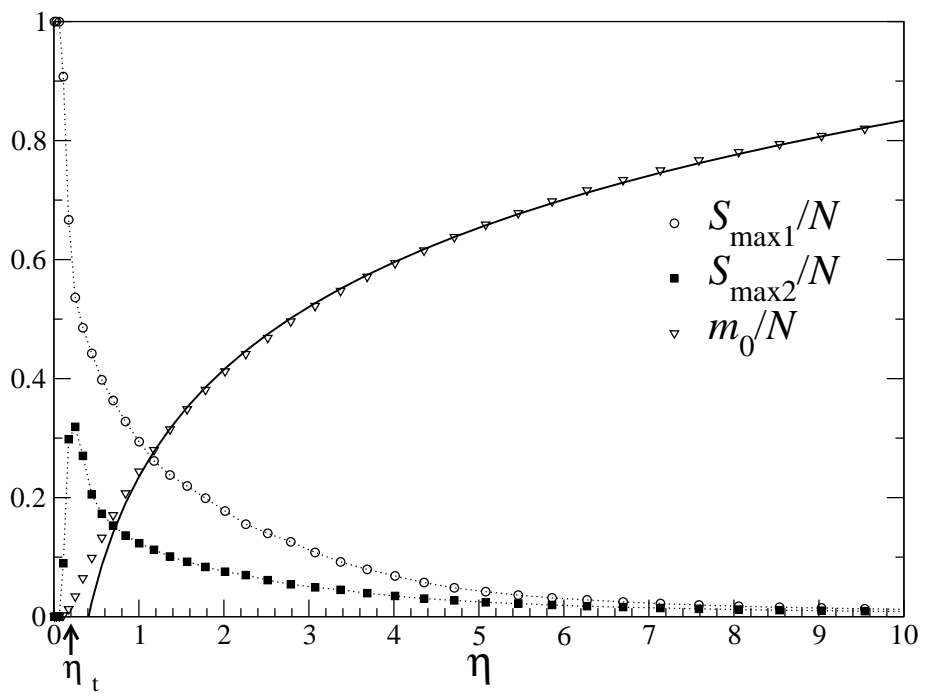

Figure 1: The mean size of the first and second largest fragments, $S_{\max 1} / N$ and $S_{\max 2} / N$, and the total number of fragments $m_{0} / N$ as functions of $\eta$ at time $t=100$. The full line corresponds to a logarithmic fit (see eq. (3)). Dotted lines are a guide for the eyes. The threshold energy $\eta_{t}$ is indicated by an arrow.

obtained in the previous article [23]. This is due to the fact that, in the present paper, we use a more rigorous method [27] for fitting.

Similar to the size of the largest fragment, the total number of fragments $m_{0}=\sum_{s} n(s)$ is a measure of the degree of break-up in the fragmentation process. Figure 1 displays also $m_{0}$ as a function of $\eta$. As expected, the total number of fragments increases with the initial kinetic energy. Moreover, $m_{0}$ presents a linear behaviour with $\eta$ for $\eta \leq 1$, and a logarithmic behaviour for $\eta \geq 1$ :

$$
\frac{m_{0}}{N}=\alpha_{m_{0}} \ln \eta+\beta_{m_{0}}
$$

where $\alpha_{m_{0}}=0.26 \pm 0.01$, i.e., the same coefficient as for $\tau$, and $\beta_{m_{0}}=0.23 \pm 0.01$. Note that Behera et al. 20] found the same linear and logarithmic behaviours while studying the impact fragmentation of a disc with a more sophisticated model, that takes into account also the elastic, shear and torque interactions between particles.

\section{Damage}

The fragment size distribution discussed in the previous section reflects the degree of damage caused by the impact. Furthermore, the increase of the total number of fragments with the impact velocity would be, and is, a direct and intuitive representation of increasing damage. However, both of these are macroscopic quantities. The microscopic level of the process is of greater interest if we want to understand how the fragmentation occurs. In our model, cracks and fragments are formed by the breaking of bonds between neighboring particles. This is illustrated in the snapshots of Fig. 3, where the bonds that 


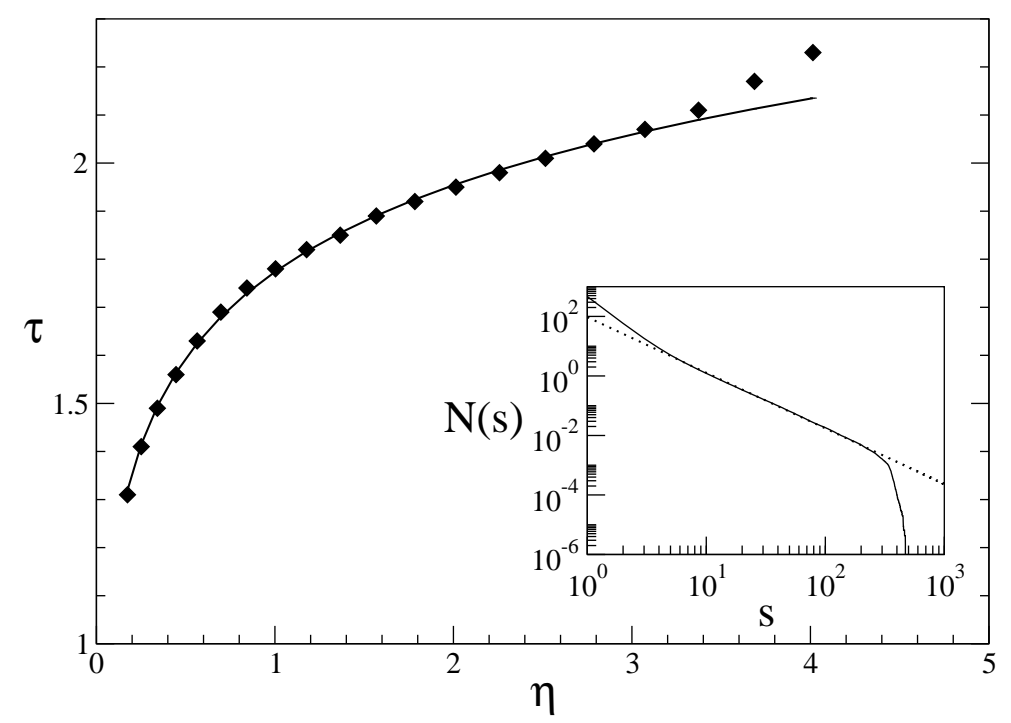

Figure 2: Power law exponent $\tau$ as a function of $\eta$. The full line is a logarithmic fit (see eq. (2) ). Cumulative fragment size distribution for $V=3(\eta=1.57)$ at $t=100$ is plotted in the inset (the dotted line is a power law function with $\tau=1.87)$

still exist at the end of the fragmentation process $(t=100)$ are shown in their initial locations before the impact. Fragments can be discerned as clusters of the remaining bonds (except the monomers, of course).

At low initial kinetic energy $(V=1, \eta=0.17)$, the disc suffers mainly from internal damage. Some internal bonds are broken and a few fragments are formed, mainly monomers in the impact zone (bottom of the figure). Oblique lines of broken bonds starting from the impact zone can be seen. As the energy deposited in the system increases $(V=2, \eta=0.70)$, oblique cracks propagate through the system from the impact zone to the edge of the disc and fragmentation in the proper sense of the word occurs. The largest fragment is localized in the top part of the disc. Most of the energy is dissipated in the impact zone, producing quantities of monomers. At higher energies $(V \geq 3, \eta \geq 1.57)$, cracks perpendicular to the former oblique ones form and create smaller fragments by merging. As energy increases further, fragments become smaller but are of various sizes, in qualitative agreement with a power law fragment size distribution. It is interesting to note, that the shape of the fragments is irregular and rough without memory of the underlying triangular lattice structure of the disc. To conclude, the crack patterns shown in Fig. 3 are consistent with the ones obtained with more complex models [17, 20].

To quantify the damage produced during the impact fragmentation, Thornton and his collaborators [17] defined the damage ratio $D(t)$ as the average ratio of the number of broken bonds at a given time $t$, to the total number of initial bonds in the disc. In the inset of Fig. 4 we plot the damage ratio for various impact velocities. At the moment of impact $(t=0), D(t)$ first increases dramatically, then at a slower rate, and eventually tends to its final value $D_{f}$, showing that the fragmentation process reaches a steady state. In fact, at later 


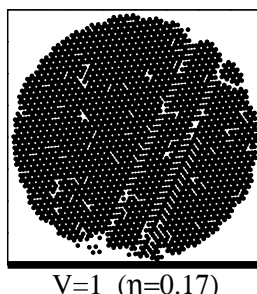

$\mathrm{V}=1 \quad \eta=0.17)$

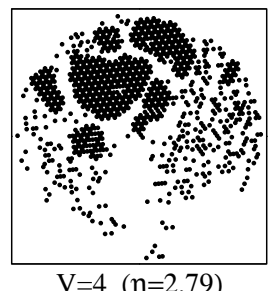

$\mathrm{V}=4 \quad(\eta=2.79)$
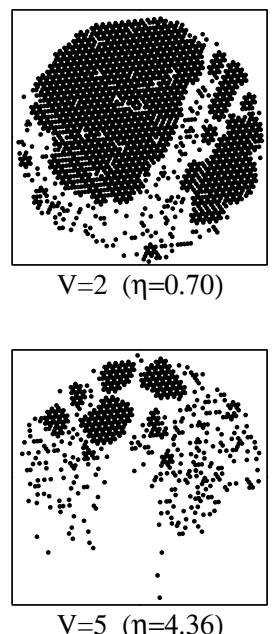
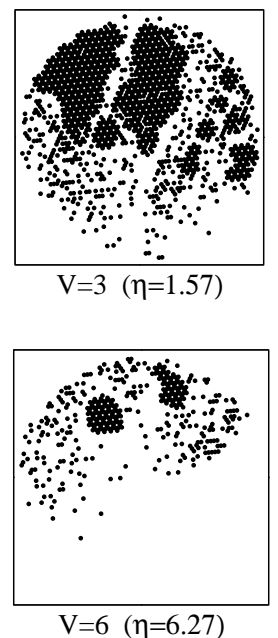

Figure 3: Snapshots at the end of the fragmentation process $(t=100)$ for various impact velocities and the same initial rotation angle. Each dot represents a bond between two particles localized at its initial position before the impact. The horizontal wall is depicted at the bottom of the top left figure.

times $(t>50)$, only few particles evaporate from exited fragments, increasing very slightly the damage ratio.

In order to better understand the evolution of damage, we calculated the damage rate, $\frac{d D(t)}{d t}$. As shown in Fig 4 the damage rate increases drastically, reaches a maximum, and then decreases slowly to zero.

This evolution is further illustrated by the snapshots in Fig 5 of the fragmenting disc for the impact velocity $V=3(\eta=1.57)$. Just after the impact, at $t_{1}=0.15$, two oblique cracks start to propagate from the impact zone. At $t_{2}=0.35$, damage increases in the impact zone and the oblique cracks become wider and continue their advance through the disc. As a consequence the damage rate increases swiftly. When the two cracks have reached the edge of the disc, the damage rate is maximal. Then, cracks perpendicular to the oblique ones are formed $\left(t_{3}=0.55\right)$. Damage spreads into the disc and damage rate decreases $\left(t \geq t_{3}\right)$. The local minimum of the damage rate $\left(t_{2} \leq t \leq t_{3}\right)$ is due to a sudden increase of the kinetic energy per particle (not shown) when the disc rebounds, after being compressed (this is observed also for higher impact velocities, see the curve for $V=5$ ). Finally, bond breakings create fragments whose surfaces evolve by evaporating particles $\left(t \geq t_{5}=1.55\right)$. As can be seen by comparing Fig. [5 at $t_{6}=10$ and Fig. [3] at $t=100$, fragments already have their overall shape at $t=10$.

As illustrated by the curves in Fig. 4, as the impact velocity increases, the damage rate increases faster and reaches a maximal value when the oblique cracks have propagated through the system. The position of the peak depends only slightly on the energy deposited in the system, while it is mostly determined by the direction of propagation of the cracks. Indeed, as the speed of sound in the disc is estimated to be around 100 [23], a crack of length $40 \sigma$ (the diameter of the disc) reaches the edge at time $t \simeq 0.4$. This is in agreement with the 


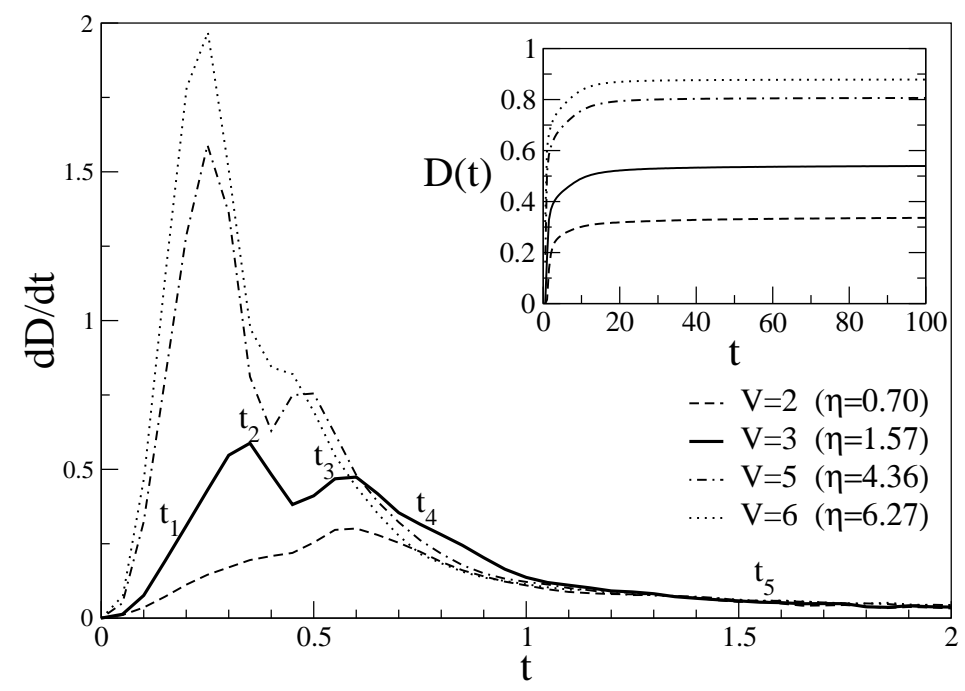

Figure 4: Damage rate $\left(\frac{d D}{d t}\right)$ as a function of time for various impact velocities. The times $t_{i}$ along the curve for $V=3$ correspond to the snapshots of Fig. 5 . In the inset, $D(t)$ as a function of time for the same impact velocities.

position of the peaks in Fig. 4. Note that the regime of evaporation is reached at the same time, around $t \simeq 1$, regardless of the energy deposited in the system.

Having studied the evolution of damage, we discuss how the final damage ratio $D_{f}$ at the end of the process behaves as a function of the initial kinetic energy. As illustrated by the curve in Fig. 6] the final damage ratio $D_{f}$ is very well fitted by a logarithmic function for $0.4 \leq \eta \leq 6$, or equivalently for $0.2 \leq D_{f} \leq 0.9$

$$
D_{f}=\alpha_{D_{f}} \ln \frac{\eta}{\eta_{t}}
$$

Here $\alpha_{D_{f}}=0.256 \pm 0.005$, i.e., almost the same coefficient as for $\tau$ and for $m_{0} / N$. For higher energies $(\eta \geq 6), D_{f}$ grows more slowly than the logarithmic function, reflecting a lower efficiency of the fragmentation process. Therefore, even at very high initial kinetic energies, some small fragments still remain. For instance, at $V=8(\eta=11.15), S_{\max 1} \simeq 13$.

The same logarithmic behaviour of $D_{f}$ was reported by Thornton et al. [17 and Behera et al. [20. For the former model, they found $\alpha_{D_{f}} \simeq 0.1$, depending on the surface energy of the disc. For the latter one, $\alpha_{D_{f}} \simeq 0.15$, as can be estimated from Fig. 8 of reference [20].

According to equations (2), (3), and (4), the power law exponent $\tau$, the total number of fragments $m_{0} / N$, and the damage ratio $D_{f}$ all exhibit a logarithmic dependence on $\eta$ with the same coefficient $\alpha$, in a certain range of energy. Consequently, we expect a linear relation between $\tau$ and $D_{f}$, and between $m_{0} / N$ and $D_{f}$. This is plotted in the inset of Fig. 6. A linear fit gives

$$
\tau=D_{f}+1.35
$$

for $0.2 \leq D_{f} \leq 0.7$ and

$$
m_{0} / N=0.96 D_{f}-0.16
$$



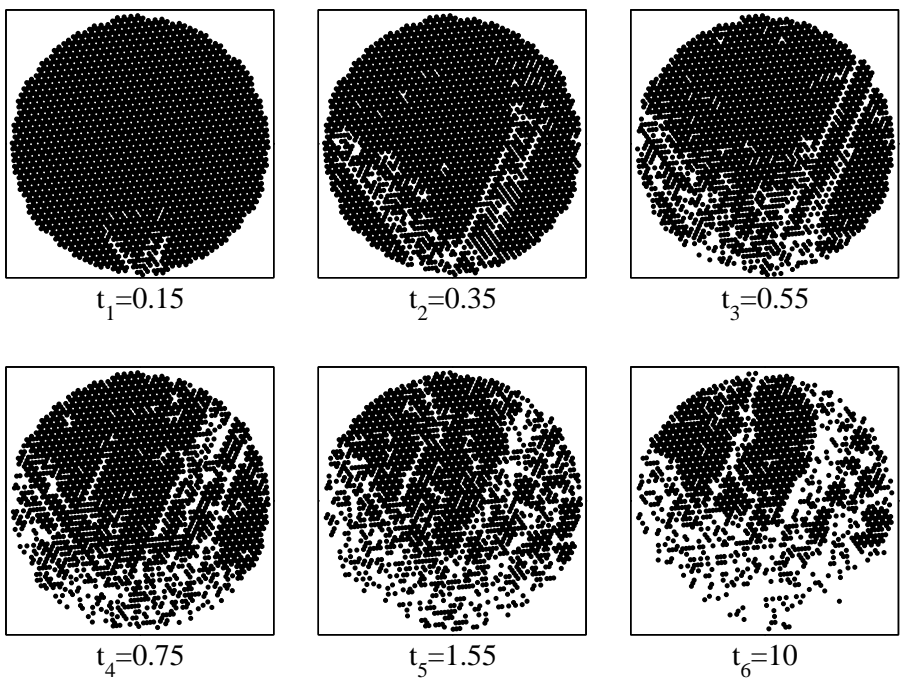

Figure 5: Snapshots at various times for $V=3(\eta=1.57)$. Each dot represents a bond between two particles placed at its initial position before the impact.

for $0.2 \leq D_{f} \leq 0.9$, with a slope slightly less than 1 . Note that using the data presented by Behera et al. [20, we found a linear relation between $m_{0}$ and $D_{f}$ as well.

\section{$5 \quad$ Fragmentation energy}

A large part of the total energy consumed in industry is used to achieve size reduction of materials. To estimate the efficiency of the fragmentation process studied in this article, we calculated the fragmentation energy. It follows from the conservation of energy that

$$
e_{p o t 0}+\frac{1}{2} m V^{2}=e_{p o t}+e_{k i n}
$$

where $e_{\text {pot }}$ and $e_{k i n}$ are the average potential and kinetic energies per particle at the end of the fragmentation process, and $e_{\text {pot } 0}=-2.87 \epsilon$ is the initial potential energy per particle. In other words, the initial kinetic energy is used to break bonds and create fragments (potential energy) and to move the fragments apart (kinetic energy). Consequently, we define the fragmentation energy $e_{f r a g}$ as the potential energy needed to form the fragments: $e_{f r a g}=e_{\text {pot }}-e_{\text {pot } 0}$.

It is easy to see that the fragmentation energy is related to the final damage ratio $D_{f}$. Using a linear fit, we found for $D_{f} \geq 0.1$ :

$$
e_{\text {frag }}=2.75 D_{f}+0.12 \text {. }
$$

Due to surface effects, the fragmentation energy is lower than $-e_{\text {pot0 }} D_{f}$ when the disc is broken into fragments. When the disc is completely shattered and $D_{f}=1$, we have $e_{f r a g}=-e_{\text {pot } 0}$, as expected.

The efficiency of the fragmentation process can be estimated by calculating the fraction of energy used to create the fragments, that is, $e_{f r a g} / e_{k i n 0}$ where 


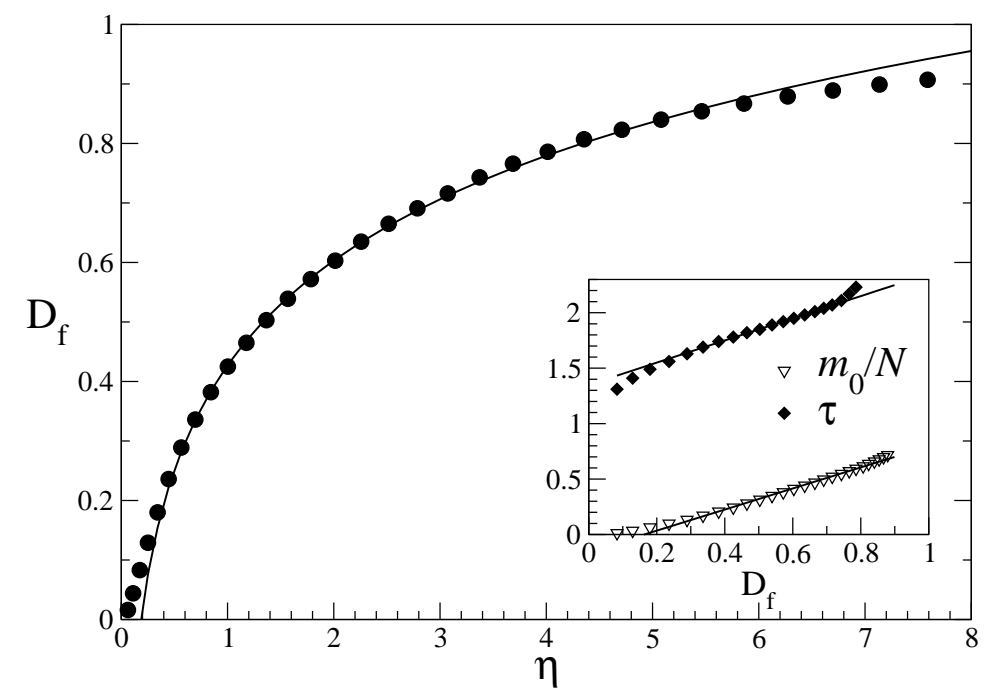

Figure 6: Final damage ratio $D_{f}$ at $t=100$ as a function of $\eta$. The full line is a logarithmic fit (see eq. (4)). The power law exponent $\tau$ and the total number of fragments $m_{0} / N$ are plotted in the inset as functions of damage ratio $D_{f}$ (the full lines are linear fits).

$e_{k i n 0}=\frac{1}{2} m V^{2}$ is the initial kinetic energy per particle. This quantity is plotted in Fig. 7 as a function of $\eta$ in the fragmentation regime, i.e., for $\eta>\eta_{t}$. The percentage of the initial kinetic energy that is used to form the fragments decreases from $70 \%$ to $10 \%$ as $\eta$ increases from $\eta_{t}$ to 10 . At high impact velocities, most of the energy is then spent on moving the fragments apart.

\section{Conclusion}

The model we propose is generic in the sense that it contains the essential physical features for investigating fragmentation phenomena. Its simplicity rests on a basic cohesive interaction between circular particles in two dimensions. Furthermore, molecular dynamics calculations allow us to determine the physical quantities involved and moreover, to study their evolution during the fragmentation process.

In this article, we have investigated the damage inflicted on a disc of interacting particles as the disc fragments upon impact with a wall - the damage being defined as the percentage of broken bonds between particles. Its propagation into the disc evidently depends on the energy deposited in the system, but the damage rate was found to be independent of the impact energy shortly after the collision. Furthermore, the percentage of energy actually used to fragment the system decreases with the energy deposited in the system, reflecting the low efficiency of the fragmentation process at high impact energy.

In particular, we have shown that the power law exponent $\tau$ of the fragment size distribution, the total number of fragments, and the damage share the same logarithmic behaviour as functions of the energy deposited in the system. Damage cannot be directly measured by experiments, but the logarithmic behaviour 


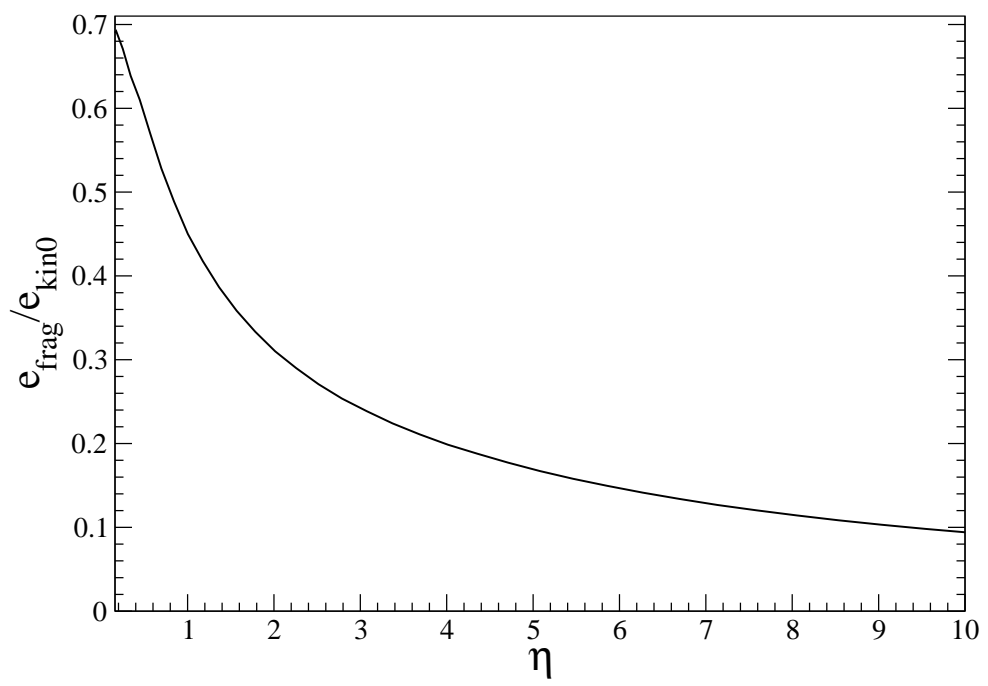

Figure 7: Fragmentation energy divided by the initial kinetic energy, $e_{\text {frag }} / e_{k i n 0}$, as a function of $\eta$, for $\eta \geq \eta_{t}$.

of $\tau$ is observed in the fragmentation of rocks [2] and liquid droplets [10. As an interesting consequence, the power law exponent and the total number of fragments are proportional to the damage. These simple relations, that we expect to be generic, may guide us to a better understanding of the underlying mechanisms of fragmentation phenomena.

\section{References}

[1] Turcotte D.L., Fractals and fragmentation, J. Geophys. Res. 91, 1921-1926 (1986)

[2] Matsui T., Waza T., Kani K. and Suzuki S., Laboratory simulation of planetesimal collision, J. Geophys. Res. 87, 10968 (1982)

[3] Meibom A. and Balslev I., Composite power laws in shock fragmentation, Phys. Rev. Lett. 76, 2492 (1996)

[4] Oddershede L., Dimon P. and Bohr J., Self-organized criticality in fragmenting, Phys. Rev. Lett. 71, 3107 (1993)

[5] Kadono T., Fragment mass distribution of platelike objects, Phys. Rev. Lett. 78, 1444 (1997)

[6] Kadono T. and Arakawa M., Crack propagation in thin glass plates caused by high velocity impact, Phys. Rev. E 65, 035107(R) (2002)

[7] Campi X., Krivine H., Sator N. and Plagnol E., Analyzing fragmentation of simple fluids with percolation theory, Eur. Phys. J. D 11, 233-238 (2000)

[8] Åström J.A., Linna R.P., Timonen J., Møller P.F. and Oddershede L., Exponential and power-law mass distributions in brittle fragmentation, Phys. Rev. E 70, 026104 (2004) 
[9] Wittel F., Kun K., Herrmann H.J. and Kröplin B.H., Fragmentation of shells, Phys. Rev. Lett 93, 035504 (2004)

[10] Moukarzel C.F., Fernández-Sabido S.F. and Ruiz-Suárez J.C., Phase transition in liquid drop fragmentation, Phys. Rev. E 75, 061127 (2007)

[11] Kaminski E. and Jaupart C., The size distribution of pyroclasts and the fragmentation sequence in explosive volcanic eruptions, J. Geophys. Res. 103, 29759-29779 (1998)

[12] Oddershede L., Meibom A. and Bohr J., Scaling analysis of meteorite shower mass distributions, Europhys. Lett. 43, 598 (1998)

[13] Holina B.L. and Grady D.E., Fragmentation by molecular dynamics: the microscopic "Big Bang", Phys. Rev. Lett. 60, 1355 (1988)

[14] Ching E.S.C., Yiu Y.Y. and Lo K.F., Energy dependence of mass distributions in fragmentation, Physica A 265, 119-128 (1999)

[15] Diehl A., Carmona H.A., Araripe L.E., Andrade J.S. Jr. and Farias G.A., Phys. Rev E 62, 4742-4746 (2000)

[16] Åström J.A., Holian B.L. and Timonen J., Universality in fragmentation, Phys. Rev. Lett. 84, 3061 (2000)

[17] Thornton C., Yin K.K. and Adams M.J., Numerical simulation of the impact fracture and fragmentation of agglomerates, J. Phys. D: Appl. Phys., 29, 424-435 (1996)

[18] Mishra B.K. and Thornton C., Impact breakage of particle agglomerates, Int. J. Miner. Process. 61, 225-239 (2001)

[19] Kun F. and Herrmann H.J., Transition from damage to fragmentation in collision of solids, Phys.Rev. E 59, 2623-2632 (1999)

[20] Behera B., Kun F., McNamara S. and Herrmann H.J., Fragmentation of a circular disc by impact on a frictionless plate, J. Phys.: Condens. Matter, 17 S2439 (2005)

[21] Wittel F.K., Carmona H.A., Kun F. and Herrmann H.J., Mechanisms in impact fragmentation, Int. J. Fract. 154, 105 (2008)

[22] Myagkov N.N. and Shumikhin T.A., Critical behavior and energy dependence of mass distributions in impact fragmentation, Physica A 358, 423$436(2005)$

[23] Sator N., Mechkov S. and Sausset F., Generic behaviours in impact fragmentation, Europ. Phys. Lett. 81, 44002 (2008)

[24] Verlet L., Computer experiments on classical fluids. I Thermodynamical properties of Lennard-Jones molecules, Phys. Rev. 159, 98 (1967)

[25] Frenkel D. and Smit B., Understanding molecular simulation: From algorithms to Applications, Academic Press, Oxford, (2001)

[26] Sator N., Clusters in simple fluids, Phys. Rep. 376, 1 (2003) 
[27] Clauset A., Shalizi C.R. and Newman M.E.J., Power-law distributions in empirical data, SIAM Review, to appear (2009) (preprint at arXiv:0706.1062) 